\title{
Coronary CT angiography in the elderly
}

\author{
R. Nijveldt • T. Pflederer $\cdot$ S. Achenbach
}

Published online: 23 August 2013

(C) The Author(s) 2013. This article is published with open access at Springerlink.com

\begin{abstract}
People over 75 years of age represent a specific group of patients for which the clinician is often in doubt about what to do, whether additional diagnostic workup is helpful or a primary medical approach would suffice. However, this patient population is less prone to the long-term effects of radiation burden or contrast medium-induced nephropathy, and therefore it may be especially worthwhile to use advanced imaging techniques such as contrast-enhanced $\mathrm{CT}$ in these patients. The following cases illustrate two common diagnostic problems, in which coronary CT angiography decided the clinical course.
\end{abstract}

\section{Keywords Coronary CT angiography $\cdot$ Elderly}

In today's clinical practice, cardiac imaging is the most frequently used diagnostic investigation. Patients admitted to the chest pain unit or outpatient clinic are often no longer stratified for risk by history, auscultation and an (exercise) electrocardiogram but have to undergo at least one imaging modality to convince the doctor the right clinical decision has been made. Also, patients are being informed by the internet, multimedia and magazines such as in the cover story of Time Magazine in 2005 (5 September, pages 32-40), and claim to know which diagnostic workup is best. The increase and advances in imaging technology have led to a tremendous growth in the use of echocardiography, nuclear tests, cardiovascular

R. Nijveldt ( $\square)$

Department of Cardiology, VU University Medical Center,

De Boelelaan 1117, 1081 HV Amsterdam, the Netherlands

e-mail: r.nijveldt@cardiologie-vumc.nl

T. Pflederer $\cdot$ S. Achenbach

Department of Cardiology, Medizinische Klinik 2, University

Hospital Erlangen, Ulmenweg 18, 91054 Erlangen, Germany magnetic resonance, computed tomography $(\mathrm{CT})$, and recently the development in hybrid imaging techniques. The general opinion of this interesting progress is the concern regarding overuse of these new technologies [1, 2], particularly for imaging modalities using ionising radiation, for which multisociety documents have been defined to guard its appropriate use [3]. However, underuse is equally important, mostly generated by a lack of knowledge of the accuracy of these techniques, the fear of radiation, and the need to refine and expand current criteria for appropriate utilisation in line with the rapid adoption of the technique $[4,5]$. People over 75 years of age represent a specific group of patients for which the clinician is often in doubt as to what to do, whether additional diagnostic workup is helpful or a primary medical approach would suffice. However, this patient population is less prone to the long-term effects of radiation burden or contrast medium-induced nephropathy [6], and therefore it may be especially worthwhile to use advanced imaging techniques such as contrast-enhanced CT in these patients. The following cases illustrate two common diagnostic problems, in which coronary $\mathrm{CT}$ angiography decided the clinical course.

The first patient is a 88 -year-old woman in an extremely good state of health, admitted to the emergency department with chest pain during a hypertensive crisis, without any other cardiovascular risk factors, a normal echocardiogram and a non-conclusive treadmill test. The second patient is a 75-year-old male patient, followed up at the outpatient clinic for dilated cardiomyopathy with mildly reduced left ventricular (LV) function for over 15 years with a suspected new wall motion abnormality in the apex on echocardiography under impaired diagnostic image quality. The combination of dilated cardiomyopathy and a suspected new regional wall motion abnormality led to the decision to perform an invasive coronary angiography. However, the patient refused an invasive procedure. 
Fig. 1 Coronary CT angiography with multiplanar reconstructions of the two described patients. The coronaries of the 88-year-old female are displayed in panels a (right coronary artery), b (left anterior descending coronary artery) and $\mathbf{c}$ (left circumflex coronary artery). The lower panels demonstrate the right coronary artery (d), the left anterior descending coronary artery (e) as well as the circumflex coronary artery (f) with mixed plaque burden of the 75-year-old male
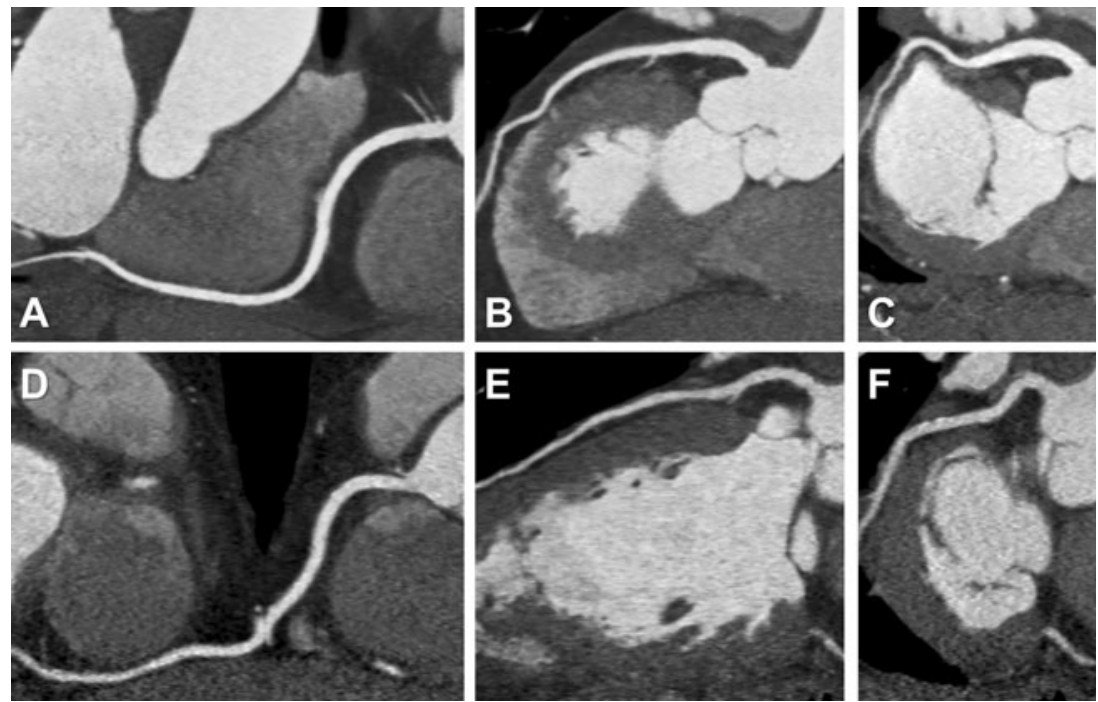

In both patients, coronary $\mathrm{CT}$ angiography with helical scanning was chosen to evaluate the coronary arteries - primarily to rule out significant coronary obstruction - and to determine LV function for further medication adjustments. The first scan was acquired with an estimated effective radiation dose of $3.5 \mathrm{mSV}$ (Figure 1, panels A-C), demonstrating no calcium and normal coronary arteries, normal LV function and mild LV hypertrophy. The woman was treated with antihypertensive medication and sent back to her general practitioner. The second scan was performed resulting in an estimated effective radiation dose of $5.2 \mathrm{mSv}$ (Figure 1, panels D-F). It showed mild, and mainly mixed, plaque burden but no significant stenoses. Furthermore, 4D reconstruction showed a normal LV function with normal systolic contractions and dimensions of the cardiac compartments. Also in this patient, there was no necessity for extension of cardiological follow-up.

These cases demonstrate that $\mathrm{CT}$ can offer an accurate, fast and complete cardiac assessment in the absence of extensive calcified coronary arteries, particularly at higher age in which radiation burden is of less importance. It is a robust alternative imaging tool for risk stratification and clinical decision making, and to dismiss or even end cardiological follow-up in the elderly patient.

\section{Funding None.}

Conflict of interests None declared.
Open Access This article is distributed under the terms of the Creative Commons Attribution License which permits any use, distribution, and reproduction in any medium, provided the original author(s) and the source are credited.

\section{References}

1. Picano E. The risks of inappropriateness in cardiac imaging. Int $\mathbf{J}$ Environ Res Public Health. 2009;6:1649-64.

2. de Boer MJ, van der Wall EE. Choosing wisely or beyond the guidelines. Neth Heart J. 2013;21:1-2.

3. Hendel RC, Patel MR, Kramer CM, et al. ACCF/ACR/SCCT/ SCMR/ASNC/NASCI/SCAI/SIR 2006 appropriateness criteria for cardiac computed tomography and cardiac magnetic resonance imaging: a report of the American college of cardiology foundation quality strategic directions committee appropriateness criteria working group, American college of radiology, society of cardiovascular computed tomography, society for cardiovascular magnetic resonance, American society of nuclear cardiology, North American society for cardiac imaging, society for cardiovascular angiography and interventions, and society of interventional radiology. J Am Coll Cardiol. 2006;48:1475-97.

4. Murphy MK, Brady TJ, Nasir K, et al. Appropriateness and utilization of cardiac CT: implications for development of future criteria. $\mathrm{J}$ Nucl Cardiol. 2010;17:881-9.

5. Weustink AC, de Feyter PJ. The role of multi-slice computed tomography in stable angina management: a current perspective. Neth Heart J. 2011;19:336-43.

6. Laugharne MJ, Paravasthu M, Preston A, et al. CT pulmonary angiography in elderly patients: outcomes in patients aged $>85$ years. Clin Radiol. 2012. doi:10.1016/j.crad.2012.09.010. 